\title{
A planetary system and a highly eccentric brown dwarf around the giant stars HIP 67851 and HIP 97233*
}

\author{
M. I. Jones ${ }^{1}$, J. S. Jenkins ${ }^{2}$, P. Rojo ${ }^{2}$, C. H. F. Melo ${ }^{3}$, and P. Bluhm ${ }^{4}$ \\ ${ }^{1}$ Department of Electrical Engineering and Center of Astro-Engineering UC, Pontificia Universidad Católica de Chile, \\ Av. Vicuña Mackenna 4860, 782-0436 Macul, Santiago, Chile \\ e-mail: mjones@aiuc.puc.cl \\ 2 Departamento de Astronomía, Universidad de Chile, Camino El Observatorio 1515, Las Condes, Casilla 36-D Santiago, Chile \\ 3 European Southern Observatory, Casilla 19001 Santiago, Chile \\ ${ }^{4}$ Departamento de Astronomía, Universidad de Concepción, Casilla 160-C Concepción, Chile
}

Received 7 August 2014 / Accepted 25 September 2014

\begin{abstract}
Context. So far more than 60 substellar companions have been discovered around giant stars. These systems present physical and orbital properties that contrast with those detected orbiting less evolved stars.

Aims. We are conducting a radial velocity survey of 166 bright giant stars in the southern hemisphere. The main goals of our project are to detect and characterize planets in close-in orbits around giant stars in order to study the effects of the host star evolution on their orbital and physical properties.

Methods. We have obtained precision radial velocities for the giant stars HIP 67851 and HIP 97233 that have revealed periodic signals, which are most likely induced by the presence of substellar companions.

Results. We present the discovery of a planetary system and an eccentric brown dwarf orbiting the giant stars HIP 67851 and HIP 97233, respectively. The inner planet around HIP 67851 has a period of 88.8 days, a projected mass of $1.4 M_{\mathrm{J}}$ and an eccentricity of 0.09 . HIP $67851 b$ is one the few known planets orbiting a giant star interior to $0.5 \mathrm{AU}$. Although the orbit of the outer object is not fully constrained, it is likely a super-Jupiter. The brown dwarf around HIP 97233 has an orbital period of 1058.8 days, a minimum mass of $20.0 M_{\mathrm{J}}$ and an eccentricity of 0.61 . This is the most eccentric known brown dwarf around a giant star.
\end{abstract}

Key words. techniques: radial velocities - brown dwarfs - planet-star interactions

\section{Introduction}

Among the currently known ensemble of exoplanets, only a small fraction have been detected around giant stars, mainly because most of the radial velocity (RV) surveys have targeted solar-type stars thus far. However, such planets have properties that are very different from those orbiting main-sequence stars. In particular, giant planets are more common ( $5 \%$ for MS host stars versus $\sim 10 \%$ for giant host stars; Döllinger et al. 2009), there are few of them in close-in orbits $(a \lesssim 0.6 \mathrm{AU}$; Johnson et al. 2007), and they are significantly more massive than planets orbiting less evolved stars (Jones et al. 2014). Additionally, the fraction of brown dwarfs (BD) in relatively close-in orbits around giant stars also seems to be higher compared to less evolved host stars (Mitchell et al. 2013). These peculiar properties of exoplanets around giant stars give us information regarding the role of stellar mass on the formation of substellar objects and the effect of the stellar evolution in the orbital and physical properties of these systems (see discussion in Jones et al. 2014).

In 2009, we began the EXPRESS project (EXoPlanets aRound Evolved StarS; Jones et al. 2011), aimed at detecting planets around evolved (giant) stars via precision radial velocities, and to study their orbital properties. So far, we have

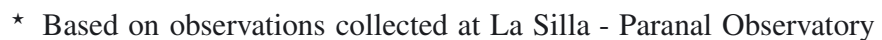
under programs ID's 085.C-0557, 087.C.0476, 089.C-0524, 090.C-0345 and through the Chilean Telescope Time under programs ID's CN 12A-073, CN 12B-047 and CN 13A-111. discovered two planetary systems (Jones et al. 2013, 2014) plus the three substellar objects presented in this paper. Interestingly, three of these are super-Jupiters and two are in relatively close-in orbits $(a \lesssim 0.6 \mathrm{AU})$.

In this paper we present a planetary system orbiting the star HIP 67851, along with the discovery of a highly eccentric brown dwarf orbiting at $\sim 2.5 \mathrm{AU}$ from the star HIP 97233 . The inner planet orbiting HIP 67851 is located at an orbital distance of $0.46 \mathrm{AU}$ whereas the outer object has a much larger separation $(a \gtrsim 4 \mathrm{AU})$. In Sect. 2, we describe the observations and the data reduction. In Sect. 3, we briefly present the radial velocity computation method. In Sect. 4, we summarize the main properties of the host stars. In Sect. 5, we present the orbital parameters of HIP $67851 b, c$ and HIP $97233 b$. Finally, in Sect. 6, we present the summary and discussion.

\section{Observations and data reduction}

During the past five years we have collected $\sim 15$ spectra for each of the 166 targets in the EXPRESS program (Jones et al. 2011, 2013), using three different high-resolution optical spectrographs installed in Chile. Among these targets, we have followed up all of them showing RV variations above the $\sim 30 \mathrm{~m} \mathrm{~s}^{-1}$ level, to determine whether they exhibit periodic variations that might be induced by orbiting planets. In particular, we took a total of 60 spectra for HIP 67851 and 41 spectra for HIP 97233, using the Fiber-fed Extended Range Optical Spectrograph (FEROS; 
Table 1. Stellar parameters of the host stars.

\begin{tabular}{lcc}
\hline \hline & HIP 67851 & HIP 97233 \\
\hline Spectral type & K0III & K0 \\
$B-V(\mathrm{mag})$ & 1.01 & 1.00 \\
$V$ (mag) & 6.17 & 7.34 \\
Parallax (mas) & $15.16 \pm 0.39$ & $9.39 \pm 0.70$ \\
$T_{\text {eff }}(\mathrm{K})$ & $4890 \pm 100$ & $5020 \pm 100$ \\
$L\left(L_{\odot}\right)$ & $17.55 \pm 2.64$ & $16.00 \pm 5.69$ \\
$\log g\left(\mathrm{~cm} \mathrm{~s}^{-2}\right)$ & $3.15 \pm 0.20$ & $3.26 \pm 0.20$ \\
{$[\mathrm{Fe} / \mathrm{H}]\left(\mathrm{dex}^{-1}\right)$} & $0.00 \pm 0.10$ & $0.29 \pm 0.13$ \\
$v \sin i\left(\mathrm{~km} \mathrm{~s}^{-1}\right)$ & $1.8 \pm 0.9$ & $2.2 \pm 0.9$ \\
$M_{\star}\left(M_{\odot}\right)$ & $1.63 \pm 0.22$ & $1.93 \pm 0.11$ \\
$R_{\star}\left(R_{\odot}\right)$ & $5.92 \pm 0.44$ & $5.34 \pm 0.55$ \\
\hline
\end{tabular}

Kaufer et al. 1999) and CHIRON (Tokovinin et al. 2013). Since these two stars are relatively bright, we obtained a signal-tonoise $\gtrsim 100$ with relatively short exposure times ( $\sim 5$ and $15 \mathrm{~min}$ for FEROS and CHIRON, respectively). The FEROS spectra were extracted using the FEROS data reduction system, while for CHIRON we used the reduction pipeline that is provided for the users. These two pipelines perform a standard reduction of echelle spectra (bias subtraction, flat-fielding correction, order tracing, extraction and wavelength calibration). For more details, see Jones et al. $(2013,2014)$.

\section{Radial velocities}

The method used to extract the radial velocities has been explained in detail in previous papers (Jones et al. 2013, 2014). Since we have used different instruments, we had to apply two different methods, namely, the simultaneous calibration method (Baranne et al. 1996) for FEROS data and the $\mathrm{I}_{2}$ cell technique (Butler et al. 1996) for CHIRON spectra. In the former case, we computed the cross-correlation function $(\mathrm{CCF})$ between the observed spectra and a template, which corresponds to a high signal-to-noise ratio observation of the same star. This procedure is repeated independently for $\sim 50 \AA$ chunks. We corrected the resulting velocity by the nightly drift, which is computed by cross-correlating the simultaneous calibration spectrum that is recorded in the sky fiber. Finally, we add the barycentric correction, which leads to the total final radial velocity at each observational epoch. The typical precision that we achieved for our targets is $\sim 5-7 \mathrm{~m} \mathrm{~s}^{-1}$ (although it is possible to reach $\sim 3 \mathrm{~m} \mathrm{~s}^{-1}$ in bright objects. See Jones et al. 2013). For the $\mathrm{I}_{2}$ cell observations, we modeled the observed spectrum of the star, which also contains the $\mathrm{I}_{2}$ absorption spectrum, in a similar fashion as presented in Butler et al. (1996). However, we use a simpler model for the instrumental point-spread-function, including only three Gaussians (a central profile of fixed width and varying height and two flanking profiles with varying height and width) and we applied this method to $5 \AA$ chunks. Although our model was optimized for the old Fiber Echelle spectrograph (which was replaced by CHIRON), it leads to a precision of $\sim 5 \mathrm{~m} \mathrm{~s}^{-1}$ for CHIRON data, which is well below the RV noise introduced by stellar pulsations in giant stars (e.g., Sato et al. 2005).

\section{Stellar properties}

The main stellar properties of HIP 67851 and HIP 97233 are listed in Table 1. The colors and parallaxes, as well as their spectral type, were taken from the HIPPARcos catalog (Van Leeuwen 2007). The atmospheric parameters of these two

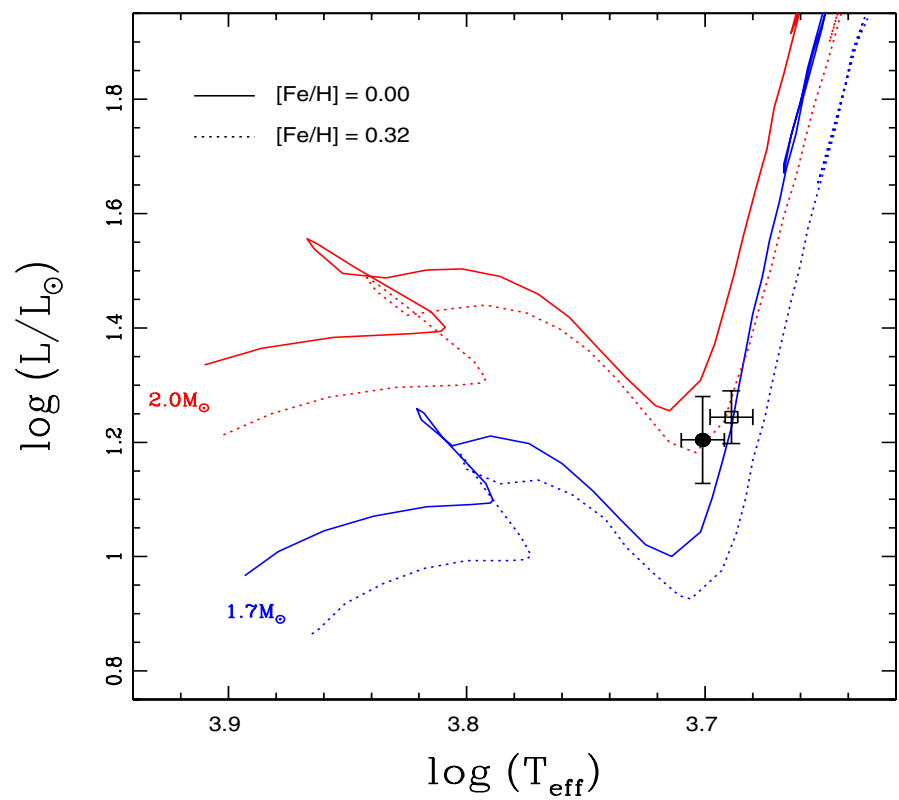

Fig. 1. Position of HIP 67851 (open square) and HIP 97233 (filled circle) in the HR diagram. Different evolutionary tracks (Salasnich et al. 2000) for $1.7 M_{\odot}$ and $2.0 M_{\odot}$ are overplotted. The solid and dotted lines correspond to $[\mathrm{Fe} / \mathrm{H}]=0.0$ and $[\mathrm{Fe} / \mathrm{H}]=0.32$, respectively.

stars were computed using the MOOG code (Sneden 1973), by matching the curve of growth of $\sim 150 \mathrm{Fe}$ I and $\sim 20 \mathrm{Fe}$ II weak lines, as explained in Jones et al. (2011). Figure 1 shows the position of HIP 67851 (open square) and HIP 97233 (filled circle) in the HR diagram. For comparison, two evolutionary tracks for $1.7 M_{\odot}$ and $2.0 M_{\odot}$ from Salasnich et al. (2000) models are overplotted, which are the closest to the position of these stars. The solid and dotted lines correspond to $[\mathrm{Fe} / \mathrm{H}]=0.0$ and $[\mathrm{Fe} / \mathrm{H}]=$ 0.32 , respectively. As can be seen, the two stars are ascending the red giant branch (RGB), although HIP 97233 is closer to the transition region between the end of the subgiant phase and the base of the RGB. Following the procedure described in Jones et al. (2011), we derived a mass of $1.63 \pm 0.22 M_{\odot}$ and stellar radius of $5.92 \pm 0.44$ for HIP 67851. Similarly, we obtained a mass of $1.93 \pm 0.11 M_{\odot}$ and radius of $5.34 \pm 0.55 R_{\odot}$ for HIP 97233 . For comparison, we obtained masses for these two stars using the method presented in da Silva et al. (2006) ${ }^{1}$. We used the spectroscopic values given in Table 1 and we corrected the $V$ magnitudes with the $A_{V}$ values obtained from Arenou et al. (1992) extinction maps. In addition, we used the Girardi et al. (2002) models. We obtained a mass of $1.41 \pm 0.18 M_{\odot}$ and radius of $5.95 \pm 0.36 R_{\odot}$ for HIP 67851 , and a mass of $1.84 \pm 0.14 M_{\odot}$ and radius of $5.20 \pm 0.50 R_{\odot}$ for HIP 97233. In both cases, the mass and radius derived by the two methods agree within one sigma.

\section{Orbital parameters}

\subsection{HIP 67851 b, $c$}

Figure 2 shows the RV measurements of HIP 67851. The black dots and red triangles correspond to FEROS and CHIRON velocities, respectively. The RV variations are listed in Tables A.1 and A.2. The solid line represents one possible Keplerian solution with an outer planet with $M_{\mathrm{P}}=6.3 M_{\mathrm{J}}, P=2200$ days and zero eccentricity. The orbital parameters of the inner planet (HIP $67851 b$ ) are listed in Table 2. The orbital fit was computed

1 http://stev.oapd.inaf.it/cgi-bin/param 


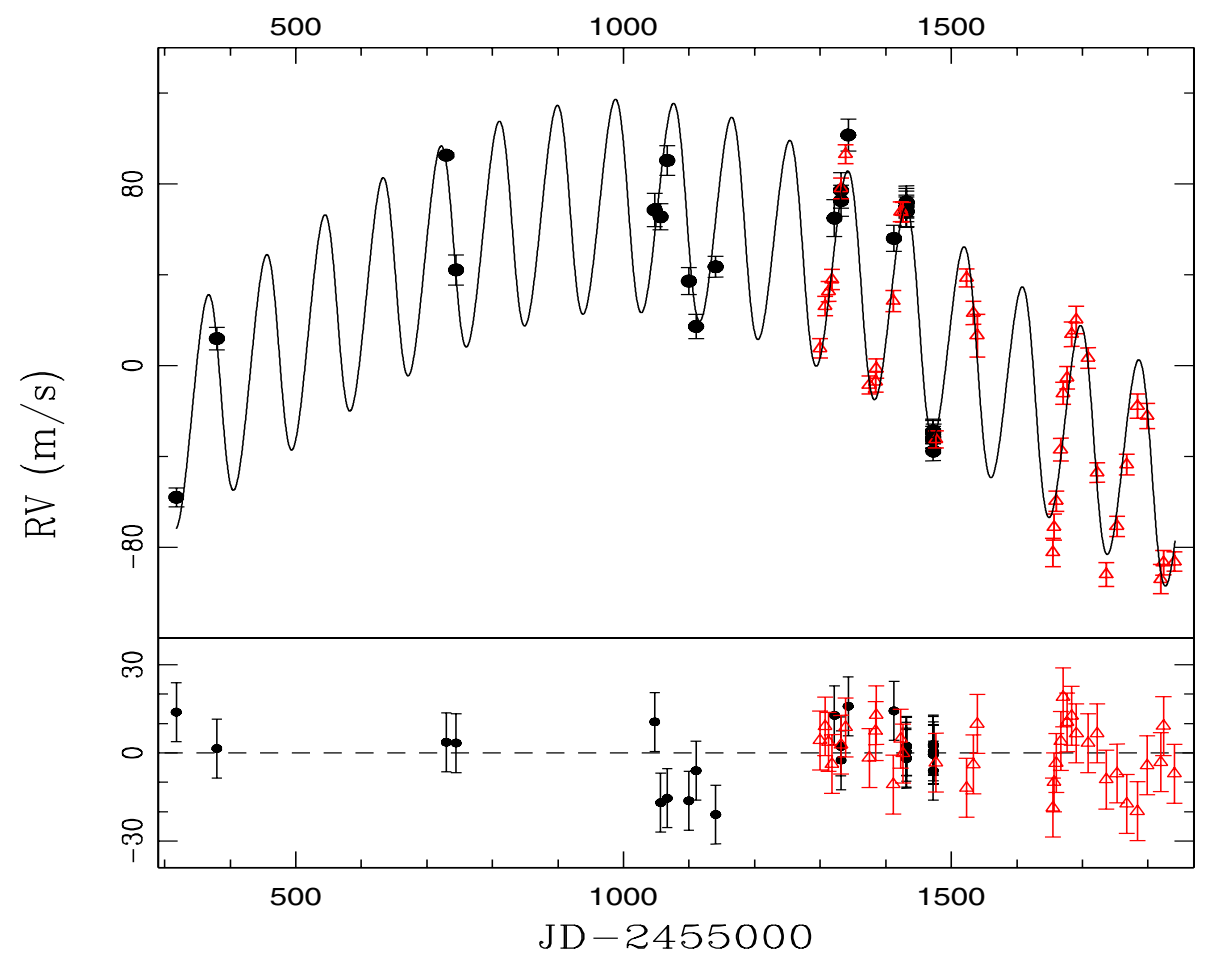

Fig. 2. Upper panel: radial velocity curve of HIP 67851. The red triangles and black dots correspond to CHIRON and FEROS velocities, respectively. One possible Keplerian solution with two giant planets is overplotted (black solid line). Lower panel: residuals from the Keplerian fit. The RMS around the fit is $9.4 \mathrm{~m} \mathrm{~s}^{-1}$.

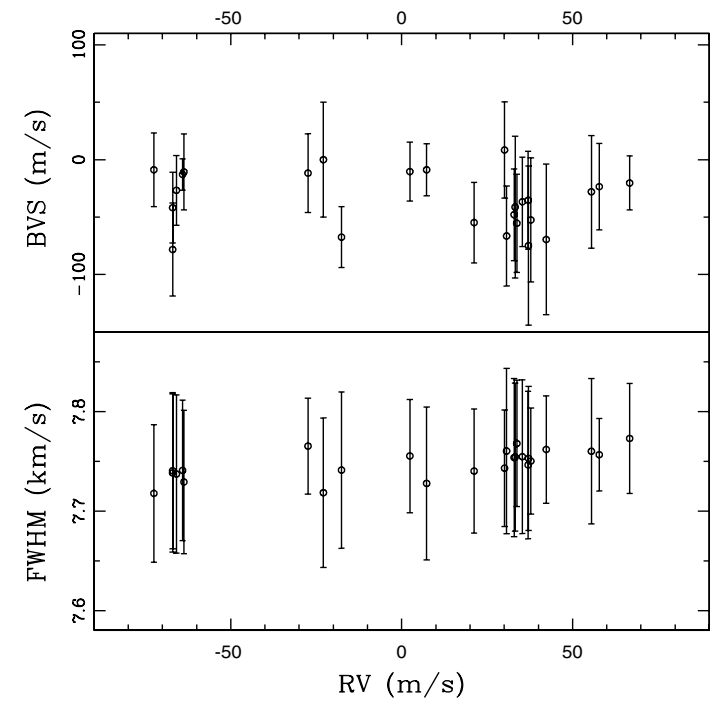

Fig. 3. Bisector velocity span and FWHM of the CCF versus FEROS radial velocities of HIP 67851 (upper and lower panel, respectively).

using the systemic console (Meschiari et al. 2009). The uncertainties were computed using a bootstrap randomization method. The RMS around the fit is $9.4 \mathrm{~m} \mathrm{~s}^{-1}$. As can be seen, although the orbital parameters of the inner planets are well determined, further follow up is needed to fully constrain the orbital period of the outer object, which probably has a mass in the planetary regime. The best Keplerian solution for HIP $67851 b$ yields an orbital distance of $0.46 \mathrm{AU}$, meaning that it is one of the few known planets orbiting interior to $0.5 \mathrm{AU}$ around giant $\mathrm{stars}^{2}$. We tested the nature of the periodic RV variations observed in HIP 67851, using the HIPPARCOS photometry of this star. This

\footnotetext{
2 Along with the transiting planets Kepler-56 b, $c$ (Huber et al. 2013), Kepler-91 $b$ (Lillo-Box et al. 2014a,b; Barclay et al. 2014) and KOI$1299 b$ (Ciceri et al. 2014; Ortiz et al. 2014). In addition, HIP $13044 b$ was shown to likely not be a real planet (Jones \& Jenkins 2014).
}

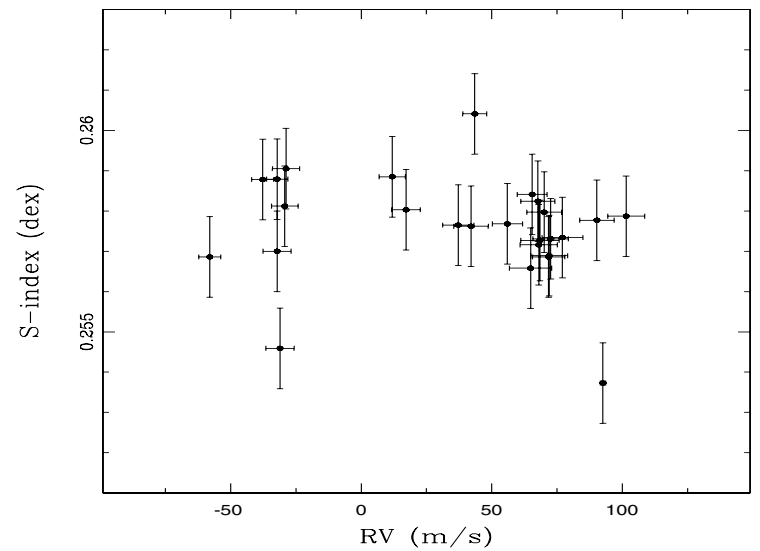

Fig. 4. Chromospheric activity indicator ( $S$-index) versus the HIP 67851 FEROS radial velocities.

dataset is comprised of 75 high-quality measurements. No significant periodic signal is observed in the data and the RMS is only 0.009 mag. In addition, we performed a line profile variation analysis. Figure 3 shows the bisector velocity span (BVS) and the full width at half maximum (FWHM) of the CCF versus the measured radial velocities (upper and lower panel, respectively). These values were computed according to the procedure explained in Jones et al. (2013). Clearly there is no correlation between these two quantities and the RV measurements. Finally, Fig. 4 shows the chromospheric activity index $(S$-index $)$ as a function of the HIP 67851 RVs. These values were computed according to the procedure described in Jenkins et al. (2008, 2011). No obvious correlation is observed between these quantities. Based on the results of these three tests, along with a failed periodogram search for any frequencies present in those data, we conclude that the RV variations observed in HIP 67851 are most likely induced by two substellar objects. 


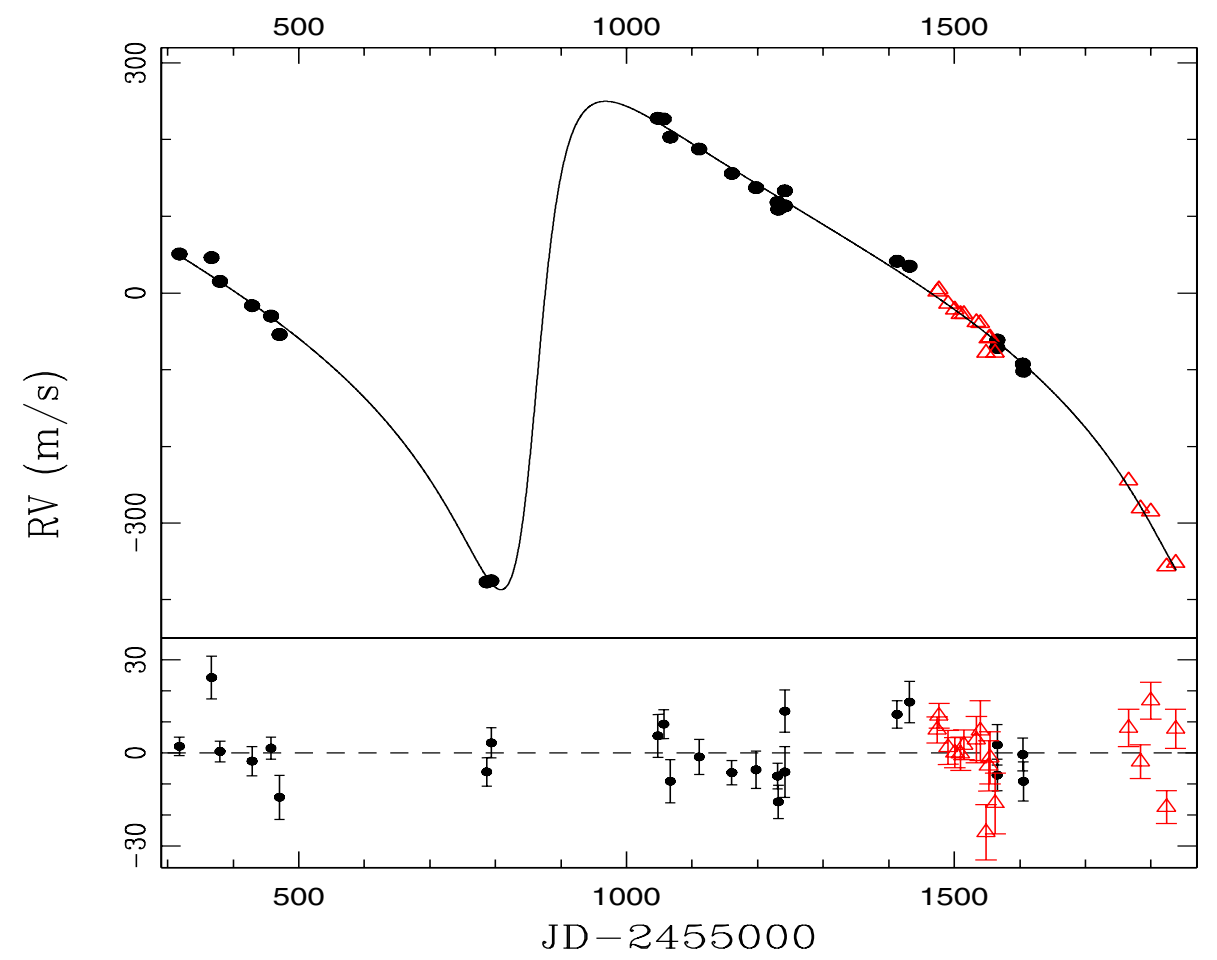

Fig. 5. Upper panel: radial velocity curve for HIP 97233. The red triangles and black dots correspond to CHIRON and FEROS velocities, respectively. The best Keplerian solution is overplotted (black solid line). Lower panel: residuals from the Keplerian fit. The RMS around the fit is $10.1 \mathrm{~m} \mathrm{~s}^{-1}$.
Table 2. Orbital parameters of HIP $67851 b$ and HIP $97233 b$.

\begin{tabular}{lcc}
\hline \hline & HIP 67851 $b$ & HIP 97233 $b$ \\
\hline$P$ (days) & $88.8 \pm 0.2$ & $1058.8 \pm 6.7$ \\
$K\left(\mathrm{~m} \mathrm{~s}^{-1}\right)$ & $46.8 \pm 2.0$ & $320.1 \pm 18.4$ \\
$a(\mathrm{AU})$ & $0.46 \pm 0.01$ & $2.55 \pm 0.05$ \\
$e$ & $0.09 \pm 0.05$ & $0.61 \pm 0.03$ \\
$\omega(\mathrm{deg})$ & $87.7 \pm 36.4$ & $249.3 \pm 2.4$ \\
$M \sin i\left(M_{\mathrm{J}}\right)$ & $1.44 \pm 0.04$ & $20.0 \pm 0.4$ \\
$T_{\mathrm{P}}(\mathrm{JD}-2455000)$ & $296.6 \pm 7.7$ & $856.3 \pm 10.0$ \\
\hline
\end{tabular}

\subsection{HIP $97233 b$}

Figure 5 shows the RV measurements of HIP 97233. The black dots and red triangles correspond to FEROS and CHIRON velocities, respectively. The solid line represents the best Keplerian fit. The orbital parameters are listed in Table 2. The RMS around the fit is $10.1 \mathrm{~m} \mathrm{~s}^{-1}$, which is consistent with the RV noise induced by stellar oscillations in giant stars (e.g., Hekker et al. 2006). The RV variations are listed in Tables A.3 and A.4.

As for HIP 67851, we investigated different possible mechanisms that might be causing the RV variations observed in HIP 97233. We analyzed the HIPPARCOS photometric data of this star, which consists of a total of 46 high quality observations. There is no significant periodic signal in the data and the RMS is below $0.01 \mathrm{mag}$. Such a low variability is not expected to mimic a signal as large as the signal observed in the HIP 97233 data (Hatzes 2002). Additionally, we performed a line profile analysis. The results from the BVS and FWHM variations are plotted in Fig. 6 (upper and lower panel, respectively). No obvious correlation is observed between these values and RV variations. Finally, Fig. 7 shows the $S$-index versus the observed radial velocities of HIP 97233. Clearly, there is no correlation with the radial velocities. Based on these results we conclude that the most likely explanation for the periodic RV variations observed in HIP 97233 is due to the presence of a substellar object.

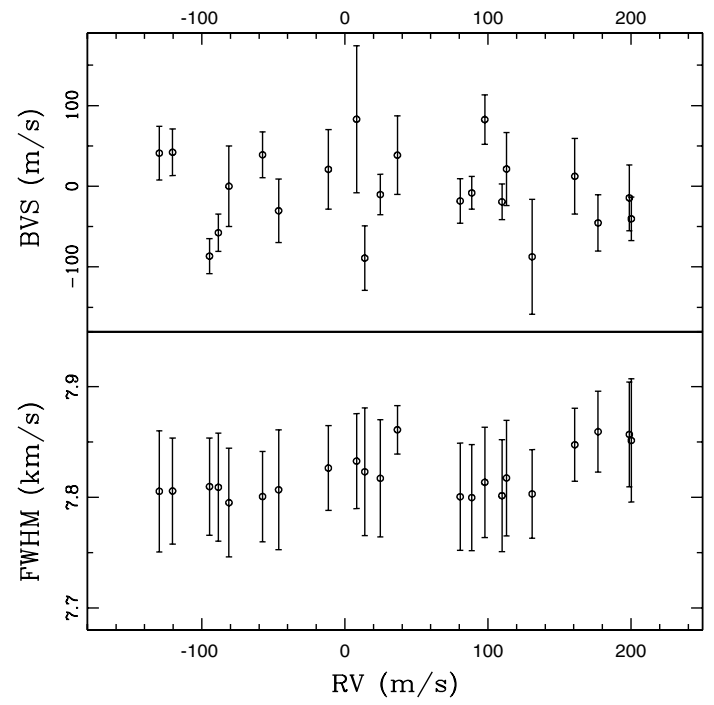

Fig. 6. Bisector velocity span and FWHM of the CCF versus FEROS radial velocities of HIP 97233 (upper and lower panel, respectively).

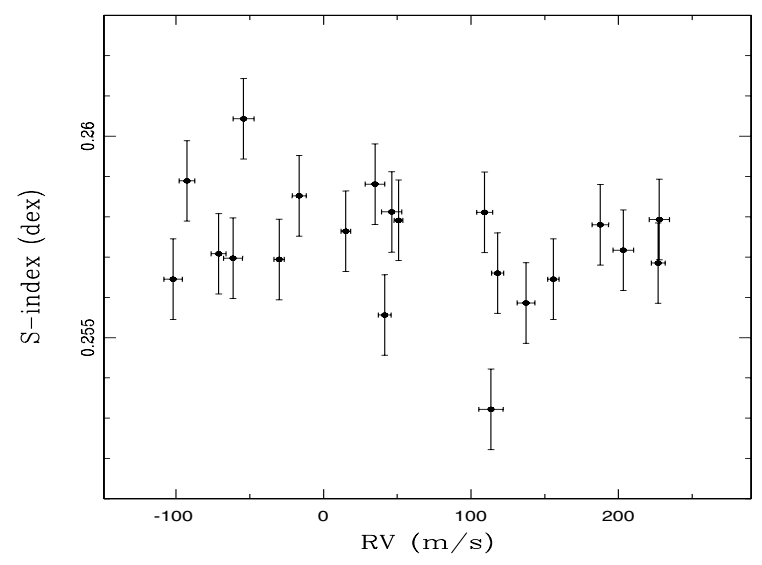

Fig. 7. Chromospheric activity indicator $(S$-index $)$ versus the HIP 97233 FEROS radial velocities. 


\section{Summary and discussion}

In this paper we present the discovery of two substellar systems found around the giant stars HIP 67851 and HIP 97233. The radial velocity variations of HIP 67851 are most likely explained by the presence of two planetary mass objects. The inner planet in the system has the following orbital parameters: $M_{\mathrm{P}} \sin i=$ $1.44 M_{\mathrm{J}}, P=88.8$ days and $e=0.09$. HIP $67851 b$ is one of the few planets orbiting interior to $\sim 0.5 \mathrm{AU}$ around giant stars. The orbital properties of the outer object are still uncertain, since only a fraction of its orbital period has been covered. However, it is probably a super-Jupiter or a BD in a wide orbit ( $a \gtrsim 4 \mathrm{AU})$. This new discovery confirms the finding by Schlaufman \& Winn (2013) and Jones et al. (2014) since HIP $67851 b$ has a nearly circular orbit $(e \lesssim 0.2)$ and a semi-major axis $\lesssim 0.9$ AU. Moreover, if HIP $67851 \mathrm{c}$ is actually a super-Jupiter, it would also confirm that most of the planets around giant stars are super-planets.

On the other hand, the RV curve of HIP 97233 shows a periodic signal that is most likely induced by a BD with the following orbital parameters: $M_{\mathrm{P}} \sin i=20.0 M_{\mathrm{J}}, P=1058.8$ days and $e=0.61$. With this new discovery, there are $\sim 10$ known BDs around giant stars, with HIP $97233 b$ being the most eccentric of these. Although this number is small, it is similar to the number of known BDs orbiting interior to $\sim 4 \mathrm{AU}$ around solar-type stars. However, since the number of giant stars targeted by RV surveys is relatively small, it seems evident that the fraction of BDs around giant stars is significantly higher than around solartype stars (Mitchell et al. 2013). The reason for this difference in the detection rate is not because of an observational bias, rather it is probably explained by either the role of the host star mass (since the giant host stars are mainly intermediate-mass stars) or by the effect of the stellar evolution. In the former case, the mass and content of the protoplanetary disk might strongly affect the efficiency of planet formation and the subsequent gas accretion. Thus, it is possible that intermediate-mass stars have more massive and dense disks, from which massive planets and BD are more efficiently formed (Lovis \& Mayor 2007). On the other hand, the mass of these super-planets might be explained by the evolution of the host star. In this scenario, a giant planet might be able to accrete a significant amount of mass from an enhanced stellar wind during the giant branch phase, eventually exceeding the deuterium burning limit and becoming a BD (Jones et al. 2014). It is also notable that the metallicity of HIP 97233 is very high $([\mathrm{Fe} / \mathrm{H}]=0.29 \pm 0.13$ dex $)$. It might thus be possible that its brown dwarf companion might have formed through core accretion (see Jenkins et al. 2009), in a process similar to what is thought to be the dominant formation mechanism for planets. If this is the case, then we would expect to detect more massive objects orbiting the most metal-rich stars since these disks form higher mass cores more efficiently, giving rise to a deficit of low-mass objects orbiting the most metal-rich stars (Jenkins et al. 2013). Finally, because of the interaction with either the disk before the gas is dissipated or with the stellar envelope during the post-MS phase, these BDs might have migrated from beyond $\sim 4 \mathrm{AU}$ to their current position. In this scenario, the primordial fraction of BDs could be the same as for solar-type host stars.

Acknowledgements. M.J. acknowledges financial support from Fondecyt project \#3140607. J.J and P.R. acknowledge funding from CATA (PB06, Conicyt). P.R. acknowledges support from Fondecyt project \#1120299 and Anillo ACT1120. We acknowledge the anonymous referee for useful comments. This research has made use of the SIMBAD database and the VizieR catalogue access tool, operated at CDS, Strasbourg, France.

\section{Appendix A: Radial velocity tables}

Table A.1. FEROS radial velocity variations of HIP 67851.

\begin{tabular}{lcc}
\hline \hline $\mathrm{JD}-2455000$ & $\mathrm{RV}\left(\mathrm{m} \mathrm{s}^{-1}\right)$ & Error $\left(\mathrm{m} \mathrm{s}^{-1}\right)$ \\
\hline 317.647 & -58.0 & 4.2 \\
379.651 & 11.9 & 5.0 \\
729.632 & 92.6 & 1.0 \\
744.602 & 42.1 & 6.6 \\
1047.622 & 68.5 & 7.3 \\
1056.609 & 65.5 & 5.7 \\
1066.625 & 90.3 & 6.6 \\
1099.610 & 37.2 & 6.0 \\
1110.584 & 17.2 & 5.4 \\
1140.607 & 43.5 & 4.6 \\
1321.799 & 64.9 & 8.1 \\
1331.734 & 77.1 & 7.8 \\
1331.806 & 72.6 & 6.8 \\
1342.780 & 101.5 & 7.1 \\
1412.574 & 56.0 & 5.8 \\
1431.540 & 67.7 & 6.5 \\
1431.587 & 70.1 & 6.7 \\
1431.640 & 68.0 & 7.1 \\
1431.686 & 71.8 & 6.2 \\
1431.750 & 72.0 & 7.1 \\
1472.606 & -29.3 & 5.1 \\
1472.610 & -32.2 & 5.4 \\
1472.627 & -28.8 & 5.2 \\
1472.643 & -37.7 & 4.2 \\
1472.657 & -32.2 & 4.1 \\
1472.712 & -31.1 & 5.4 \\
\hline & &
\end{tabular}

Table A.2. CHIRON radial velocity variations of HIP 67851.

\begin{tabular}{lcc}
\hline \hline JD -2455000 & RV $\left(\mathrm{m} \mathrm{s}^{-1}\right)$ & Error $\left(\mathrm{m} \mathrm{s}^{-1}\right)$ \\
\hline 1299.843 & 7.6 & 4.3 \\
1307.835 & 26.2 & 4.3 \\
1312.800 & 32.6 & 4.4 \\
1317.784 & 37.9 & 4.5 \\
1331.897 & 78.0 & 4.6 \\
1338.805 & 93.1 & 4.1 \\
1374.783 & -8.5 & 4.0 \\
1384.685 & -7.2 & 4.4 \\
1385.645 & -1.3 & 4.3 \\
1411.529 & 28.4 & 4.7 \\
1422.692 & 67.7 & 4.4 \\
1426.595 & 67.6 & 4.3 \\
1476.654 & -32.5 & 3.7 \\
1523.469 & 38.6 & 4.0 \\
1533.483 & 23.2 & 5.1 \\
1539.480 & 13.2 & 9.4 \\
1654.819 & -82.3 & 6.2 \\
1656.815 & -71.1 & 5.7 \\
1659.806 & -59.7 & 4.5 \\
1666.858 & -37.0 & 5.0 \\
1670.816 & -12.2 & 4.9 \\
1676.760 & -5.4 & 5.0 \\
1683.887 & 13.8 & 5.4 \\
1690.891 & 20.1 & 6.0 \\
1708.828 & 3.3 & 4.5 \\
1722.691 & -47.1 & 4.3 \\
1736.616 & -92.0 & 5.2 \\
1752.679 & -70.8 & 4.5 \\
1767.540 & -43.6 & 4.5 \\
1783.751 & -17.8 & 5.4 \\
1798.616 & -22.2 & 5.6 \\
1819.563 & -94.0 & 6.5 \\
1823.577 & -86.8 & 5.4 \\
1840.551 & -86.3 & 4.3 \\
\hline & &
\end{tabular}


Table A.3. FEROS radial velocity variations of HIP 97233.

\begin{tabular}{lcc}
\hline \hline $\mathrm{JD}-2455000$ & $\mathrm{RV}\left(\mathrm{m} \mathrm{s}^{-1}\right)$ & Error $\left(\mathrm{m} \mathrm{s}^{-1}\right)$ \\
\hline 317.821 & 50.9 & 3.0 \\
366.873 & 46.2 & 6.9 \\
379.827 & 15.1 & 3.3 \\
428.705 & -16.5 & 4.7 \\
457.619 & -30.1 & 3.5 \\
470.603 & -54.2 & 7.1 \\
786.752 & -376.7 & 4.6 \\
793.742 & -375.3 & 4.9 \\
1047.819 & 227.7 & 6.9 \\
1056.778 & 226.9 & 4.7 \\
1066.788 & 203.3 & 6.9 \\
1110.761 & 187.7 & 5.6 \\
1160.694 & 155.9 & 3.9 \\
1197.626 & 137.3 & 6.0 \\
1230.525 & 118.0 & 4.1 \\
1231.500 & 109.2 & 5.4 \\
1241.487 & 133.2 & 6.8 \\
1241.492 & 113.6 & 8.2 \\
1412.790 & 41.5 & 4.4 \\
1431.767 & 34.9 & 6.7 \\
1565.547 & -71.1 & 5.1 \\
1565.596 & -61.4 & 6.5 \\
1604.491 & -92.6 & 5.3 \\
1605.503 & -102.0 & 6.3 \\
\hline & &
\end{tabular}

\section{References}

Arenou, F., Grenon, M., \& Gómez, A. 1992, A\&A, 258, 104 Baranne, A., Queloz, D., Mayor, M., et al. 1996, A\&A, 119, 373 Barclay, T., Endl, M., Huber, D., et al. 2014, ApJ, submitted [arXiv: 1408.3149]

Butler, R. P., Marcy, G. W., Williams, E., et al. 1996, PASP, 108, 500 Ciceri, S., Lillo-Box, J., Southworth, J., et al. 2014, A\&A, in press, DOI: $10.1051 / 0004-6361 / 201425145$

da Silva, L., Girardi, L., Pasquini, L., et al. 2006, A\&A, 458, 609

Döllinger, M. P., Hatzes, A. P., Pasquini, L., Guenther, E. W., \& Hartmann, M. 2009, A\&A, 505, 1311

Girardi, L., Bertelli, G., Bressan, A., et al. 2002, A\&A, 391, 195

Hatzes, A. P. 2002, Astron. Nachr., 323, 392

Hekker, S. Reffert, S., Quirrenbach, A., et al. 2006, A\&A, 454, 943

Huber, D., Carter, J. A., Barbieri, M., et al. 2013, Science, 342, 33

Jenkins, J. S., Jones, H. R. A., Pavlenko, Y., et al. 2008, A\&A, 485, 571

Jenkins, J. S., Jones, H. R. A., Goździewski, K., et al. 2009, MNRAS, 398, 911

Jenkins, J. S., Murgas, F., Rojo, P., et al. 2011, A\&A, 531, A8

Jenkins, J. S., Jones, H. R. A., Tuomi, M., et al. 2013, ApJ, 766, 67

Johnson, J. A., Fischer, D. A., Marcy, G. W., et al. 2007, A\&A, 665, 785

Jones, M. I., \& Jenkins, J. S. 2014, A\&A, 562, A129
Table A.4. CHIRON radial velocity variations of HIP 97233.

\begin{tabular}{lcc}
\hline \hline $\mathrm{JD}-2455000$ & $\mathrm{RV}\left(\mathrm{m} \mathrm{s}^{-1}\right)$ & Error $\left(\mathrm{m} \mathrm{s}^{-1}\right)$ \\
\hline 1473.851 & 1.7 & 4.2 \\
1476.798 & 4.5 & 4.0 \\
1490.792 & -14.0 & 5.5 \\
1500.740 & -21.8 & 4.8 \\
1509.719 & -27.7 & 5.4 \\
1514.623 & -27.9 & 4.8 \\
1533.651 & -38.3 & 7.5 \\
1539.628 & -39.3 & 9.6 \\
1548.606 & -78.0 & 8.9 \\
1552.511 & -59.2 & 8.1 \\
1554.550 & -58.0 & 8.4 \\
1562.531 & -78.1 & 9.8 \\
1765.847 & -244.7 & 6.0 \\
1783.910 & -280.8 & 5.4 \\
1799.840 & -284.9 & 5.9 \\
1823.733 & -356.4 & 5.3 \\
1837.707 & -352.2 & 6.3 \\
\hline
\end{tabular}

Jones, M. I., Jenkins, J. S., Rojo, P., \& Melo, C. H. F. 2011, A\&A, 536, A71 Jones, M. I., Jenkins, J. S., Rojo, P., Melo, C. H. F., \& Bluhm, P. 2013, A\&A, 556, A78

Jones, M. I., Jenkins, J. S., Bluhm, P., Rojo, P., \& Melo, C. H. F. 2014, A\&A, 566, A113

Kaufer, A., Stahl, O., Tubbesing, S., et al. 1999, The Messenger, 95, 8

Lillo-Box, J., Barrado, D., Moya, A., et al. 2014a, A\&A, 562, A109

Lillo-Box, J., Barrado, D., Henning, Th., et al. 2014b, A\&A, 568, A1

Lovis, C., \& Mayor, M. 2007, A\&A, 472, 657

Meschiari, S., Wolf, A. S., Rivera, E., et al. 2009, PASJ, 121, 1016

Mitchell, D. S., Reffert, S., Trifonov, T., Quirrenbach, A., \& Fischer, D. A. 2013, A\&A, 555, A87

Ortiz, M., Gandolfi, D., Reffert, S., et al. 2014, A\&A, accepted [arXiv: 1410.3000 ]

Salasnich, B., Girardi, L., Weiss, A., \& Chiosi, C. 2000, A\&A, 361, 1023

Sato, B., Kambe, E., Takeda, Y., et al. 2005, PASJ, 57, 97

Schlaufman, K., \& Winn, J. 2013, ApJ, 772, 143

Setiawan, J., Klement, R. J., Henning, T., et al. 2010, Science, 330, 1642

Sliski, D. H., \& Kipping, D. M. 2014, ApJ, 788, 148

Sneden C. 1973, ApJ, 184, 839

Tokovinin, A., Fischer, D. A., Bonati, M., et al. 2013, PASP, 125, 1336

Van Leeuwen, F. 2007, A\&A, 474, 653

Wolszczan, A., \& Frail, D. A. 1992, Nature, 355, 14 1992, Nature, 355, 1455 